\title{
Supervivencia de pacientes con cáncer de cuello uterino tratadas en el Instituto Nacional de Cancerología
}

\author{
Constanza Pardo¹, Ricardo Cendales² \\ 1 Grupo de Vigilancia Epidemiológica del Cáncer, Instituto Nacional de Cancerología, Bogotá, D.C., \\ Colombia. \\ 2 Grupo de Radioterapia Oncológica, Instituto Nacional de Cancerología, Bogotá, D.C., Colombia.
}

Introducción. El cáncer de cuello uterino es el de mayor incidencia y la primera causa de mortalidad en las mujeres colombianas. Cerca de $10 \%$ de todos los casos del país son tratados en el Instituto Nacional de Cancerología. Se requiere evaluar la experiencia institucional.

Objetivo. Describir la supervivencia global a cinco años de las pacientes con cáncer de cuello uterino tratadas en el Instituto Nacional de Cancerología durante el 2000.

Materiales y métodos. Análisis de supervivencia que incluyó todas las pacientes con cáncer de cuello uterino que fueron tratadas en el Instituto Nacional de Cancerología en el 2000. Se emplearon frecuencias y medidas de tendencia central y de dispersión, para resumir las variables categóricas y numéricas, respectivamente. El análisis de supervivencia se realizó mediante el método de Kaplan-Meier y la regresión de Cox.

Resultados. Se trataron 651 pacientes en el 2000. Entre las 455 pacientes elegibles, 303 (66\%) estaban vivas al finalizar el período. El tiempo medio de seguimiento para las pacientes que no murieron fue de 3,69 años, con una desviación estándar de 2,58 años. La probabilidad acumulada de supervivencia global a 5 años fue de 58,8\% y el tiempo medio de supervivencia fue de 4,53 años. La única variable que afectó significativamente la función de supervivencia en el análisis multivariado de Cox fue el estadio clínico al momento del diagnóstico.

Conclusiones. Los resultados de supervivencia global son similares a los reportados en la literatura. Se recomienda desarrollar estudios en cohortes más grandes para aumentar la potencia del estudio e identificar otros factores de pronóstico.

Palabras clave: análisis de supervivencia, neoplasias del cuello uterino, registros de mortalidad, mortalidad hospitalaria, vigilancia epidemiológica, Colombia.

\section{Survival analysis of cervical cancer patients}

Introduction. Uterine cervical cancer is the first cause of incidence and mortality in Colombian women. Nearly $10 \%$ of all the cases in the country are treated at the Instituto Nacional de Cancerología. Evaluation of the institutional success rates is suggested.

Objective. Patient survival over a 5 year period was summarized for those admitted for treatment of cervical cancer at the Institute in the year 2000.

Materials and methods. All patients with cervical cancer at the Institute in 2000 were included in the survival analysis. Frequencies, central tendencies and dispersion measures were used to describe categorical and numerical variables. Survival analysis was performed by using Kaplan Meier and the multivariate Cox regression model.

Results. During the study period, 651 patients with cervical cancer were treated. Among the 455 eligible patients, 303 (66\%) were alive at the end of the study period. Mean survival time for patients who did not die was 3.69 years, with a standard deviation of 2.58 years. Cumulated five year overall survival probability was $58.8 \%$ and mean survival was 4.53 years. The only variable that significantly affected the survival function was the clinical stage at the time of the diagnosis. Conclusions. Overall survival results are similar to those described in other international institutions. If larger cohort studies were used, the power of the study can be increased in order to identify other factors associated with the prognosis.

Key words: survival analysis, uterine cervical neoplasms, mortality registries, hospital mortality, epidemiologic surveillance, Colombia. 
El cáncer de cuello uterino pasó del segundo al primer lugar en incidencia $(36,4$ por 100.000 habitantes) y en mortalidad (18,2 por 100.000 habitantes) por cáncer entre las mujeres colombianas en 2002. Para Suramérica, las tasas de incidencia y mortalidad para el mismo año fueron de 19,1 y 12,9 por 100.000 habitantes, respectivamente (1). Durante el periodo de 1995 a 1999, fue la primera causa de muerte relacionada con la salud sexual y procreativa de la mujer en Colombia. La tendencia de la mortalidad para el cáncer de cuello uterino en los últimos quince años se ha mantenido sin variación en Colombia, a pesar de que en 1991 se creó el programa nacional para su detección precoz (2) y que, dentro del Sistema General de Seguridad Social, las actividades para el control de esta enfermedad han sido una prioridad en salud pública $(3,4)$.

La supervivencia libre de enfermedad y de recaída local y regional entre pacientes con cáncer de cuello uterino, está determinada principalmente por el estadio clínico y la presencia de compromiso ganglionar, aunque la invasión del estroma y la presencia de invasión vascular o linfática son también factores de pronóstico para pacientes con tumores en estadios tempranos (5). Otros factores de pronóstico menos importantes son el tipo histológico y el grado de diferenciación. La edad, el nivel socioeconómico, la raza y los niveles de hemoglobina pierden su efecto al incluir los demás factores en modelos multivariados, por lo que su papel como factores de pronóstico no está plenamente establecido (6).

La supervivencia en cáncer de cuello uterino ha mostrado variaciones importantes entre los países desarrollados y los países en desarrollo, debido a que en estos últimos los programas de tamización son ineficaces, lo cual se

\footnotetext{
Correspondencia:

Constanza Pardo, Grupo de Vigilancia Epidemiológica del Cáncer, Instituto Nacional de Cancerología, Calle 1 № 9-85, Bogotá, D.C., Colombia.

Teléfono: (571) 334 1177, 334 1111, extensión 4108; fax: (571) 3341353

cpardo@cancer.gov.co, tachapardo@yahoo.com

Recibido: 24/10/08; aceptado:06/04/09
}

relaciona con los altos costos operativos que implica la infraestructura, el recurso humano, el tratamiento y el seguimiento de las mujeres con anormalidades citológicas (7); por lo tanto, en los países en desarrollo los cánceres de cuello uterino suelen diagnosticarse en estadios avanzados.

En el Instituto Nacional de Cancerología, el cáncer de cuello uterino es la primera causa de consulta por morbilidad, con un promedio de 713 casos por año; la edad media es de 46,5 años y el $98 \%$ de los casos se diagnostica por histopatología. En 2004, de los 739 casos hospitalizados por cáncer de cuello uterino, el $22 \%$ era in situ y el $34,6 \%$ fue diagnosticado en estadios I y II (8).

El Instituto Nacional de Cancerología es un centro de referencia del orden nacional y cuenta con un registro institucional de cáncer que permite identificar de manera simple y eficiente los pacientes nuevos; también, cuenta con un sistema de registro de mortalidad institucional. Los registros de base hospitalaria recopilan información sobre todos los casos nuevos de cáncer que ingresan a la institución, pueden usarse para describir la experiencia de cualquier grupo de pacientes con cáncer y permiten hacer seguimiento de la supervivencia en subgrupos específicos de población (9).

El análisis descriptivo de supervivencia es una herramienta útil para la evaluación de la efectividad de la terapia. Sin embargo, para poder hacer estimaciones válidas de la supervivencia, resulta imprescindible incluir el universo de pacientes elegibles, con el fin de no incurrir en sesgos de selección; este universo se puede recopilar a partir de los registros institucionales o de población (10).

En Colombia, no hay estudios recientes publicados en revistas indexadas que describan la supervivencia de las pacientes con cáncer de cuello uterino. A pesar de que el Instituto Nacional de Cancerología es un centro nacional de referencia que ofrece tratamiento, aproximadamente, al $10 \%$ de todos los casos de cáncer de cuello uterino en Colombia, no tiene evaluaciones periódicas de supervivencia. 
El objetivo de este estudio es describir la supervivencia global a cinco años, de las pacientes con cáncer de cuello uterino que se trataron por primera vez en el Instituto Nacional de Cancerología entre el $1^{\circ}$ de enero y el 31 de diciembre de 2000, haciendo uso de los registros institucionales de cáncer y mortalidad.

\section{Materiales y métodos}

Se incluyeron todas las pacientes que se hospitalizaron y se trataron por primera vez en el Instituto Nacional de Cancerología en el 2000, con diagnóstico de cáncer de cuello uterino hecho en la institución o por fuera de ella y con confirmación histopatológica del tumor. Se excluyeron las pacientes con antecedentes de procedimientos terapéuticos previos orientados a tratar la enfermedad neoplásica del cuello uterino, pacientes con recidivas tumorales, pacientes con cáncer de cuello uterino como segundo primario y pacientes que hubiesen recibido cualquier componente del tratamiento fuera del Instituto Nacional de Cancerología.

Las fuentes de información fueron la historia clínica, el contacto telefónico con las pacientes o familiares, las bases de datos del registro institucional y de mortalidad, y las bases de mortalidad distrital y nacional consultadas a través de la Secretaría Distrital de Salud. Para la recolección de las variables, se empleó un formato específicamente diseñado para el estudio. El seguimiento se hizo de manera retrospectiva $y$, para aquellas pacientes que no tenían la última visita actualizada, se inició el proceso de contacto telefónico con el apoyo de una trabajadora social entrenada en el seguimiento de pacientes con cáncer.

Previo a la digitalización, se depuraron los datos mediantela revisión de los formatos diligenciados, con el fin de detectar los espacios en blanco y las inconsistencias y para verificarlos con la historia clínica, si era necesario. Los datos relacionados con el tipo histológico se codificaron con la Clasificación Internacional de Enfermedades para Oncología, segunda edición (CIEO-2). La información diligenciada en el formato se digitó en una base de datos validada en Epi-Info 2002. Se realizaron análisis de consistencia y validez de la base de datos; los datos inconsistentes se corrigieron a partir de la revisión del formato de recolección y de la historia clínica.

Se calculó la variable "tiempo de observación" a partir de la fecha del primer tratamiento y la fecha del último contacto o la muerte; para aquellas pacientes que por alguna razón no recibieron tratamiento, esta variable se calculó a partir de la fecha del ingreso al Instituto. Aquellos casos en los que no se logró establecer la fecha o la causa de la defunción, se consideraron como casos censurados a la derecha; en consecuencia, su estado fue el correspondiente al del último contacto. Para aquellos casos en los que no se logró establecer la fecha de inicio del tratamiento, se hizo una imputación probabilística del tiempo transcurrido entre la fecha de inicio del tratamiento y la fecha de ingreso a la institución; este tiempo se adicionó al de la fecha de ingreso a la institución y esta fecha se asumió como la fecha de inicio de tratamiento.

Para el análisis de los datos se usó el programa SPSS, versión 13. Se realizaron análisis univariados para todas las variables del estudio. Para las variables categóricas, se calcularon razones y proporciones, mientras que para las variables cuantitativas se presentaron medidas de tendencia central y dispersión. El análisis de supervivencia global se hizo de forma descriptiva con el método de Kaplan-Meier y se empleó la prueba de rango logarítmico para comparar las funciones de supervivencia de aquellas variables que tenían más de una categoría. El análisis multivariado de factores de pronóstico se realizó con un modelo de regresión de Cox. Se verificaron los supuestos de riesgos proporcionales para cada factor y para el modelo global en el modelo definitivo; también, se verificó el ajuste del modelo, la ausencia de colinearidad y la presencia de valores atípicos influyentes. En el modelado se partió de uno jerárquico bien estructurado y se descartaron variables con el método paso a paso hacia atrás con un criterio de permanencia de 0,05.

\section{Resultados}

De acuerdo con el registro institucional de cáncer, entre el $1^{\circ}$ de enero y el 31 de diciembre de 2000, 
se atendieron 651 pacientes con cáncer de cuello uterino en el Instituto Nacional de Cancerología. Se excluyeron del análisis 196 pacientes: una, por no tener confirmación histopatológica; 109, por haber recibido tratamiento previo para el cáncer de cuello uterino antes de ingresar a la institución; 8, porque el cáncer de cuello uterino era un segundo tumor primario y 78 , porque no fueron tratadas en la institución. Los resultados se presentan para las 455 pacientes elegibles para el estudio.

De las 455 pacientes elegibles, 152 habían muerto al final del seguimiento y 303 estaban vivas al último control, por lo que se consideraron como casos censurados. Entre las 303 pacientes que estaban vivas en el último control, $153 \mathrm{se}$ habían seguido, al menos, durante cinco años. En conjunto, se logró seguir hasta la muerte o por más de cinco años a 305 (67\%) pacientes.

De las 152 pacientes que murieron a causa de la enfermedad, 128 se identificaron a partir del cruce de la base de datos del registro institucional de cáncer y las bases de datos de mortalidad institucional y nacional. El estado vital se actualizó para 131 pacientes a partir de llamadas telefónicas exclusivamente; mediante este mecanismo se identificaron 16 muertes adicionales. No se actualizó el estado vital para 12 de estos casos, dado que no se logró establecer la fecha ni la causa de la defunción; por lo tanto, se asumió que estaban vivas y, en consecuencia, fueron censurados en el seguimiento inmediatamente anterior.

\section{Características sociodemográficas}

La población de pacientes con cáncer de cuello uterino que atiende el Instituto Nacional de Cancerología es vulnerable, con un nivel escolar bajo y afiliación al régimen subsidiado (cuadro 1). Más de $80 \%$ de la población procede del distrito capital y de cinco departamentos: Bogotá (34,9\%), Cundinamarca $(19,1 \%)$, Tolima $(10,3 \%)$, Boyacá $(8,5 \%)$, Huila $(5,7 \%)$ y Casanare $(4,8 \%)$. Sin embargo, en el Instituto Nacional de Cancerología se atienden pacientes de prácticamente todo el país.

\section{Características clínicas}

La mayoría de las pacientes tenía tumores de tipo escamocelular; un poco más de la mitad tenía tumores mayores de $5 \mathrm{~cm}$ y estadios IIIB o superiores. Un porcentaje importante de las pacientes no tenía información con respecto al grado de diferenciación tumoral (cuadro 2).

\section{Características del tratamiento}

El $53,2 \%$ de las pacientes fue tratada con radioterapia exclusivamente; este porcentaje fue mayor para los estadios más avanzados. El $23 \%$ de las pacientes se trataron con cirugía exclusivamente; este porcentaje fue mayor en

Cuadro 1. Características sociodemográficas de las pacientes con cáncer de cuello uterino tratadas en el Instituto Nacional de Cancerología, 2000.

\begin{tabular}{llrr}
\hline Variable & Niveles de la variable & $\mathbf{n}$ & $\%$ \\
\hline Nivel educativo máximo alcanzado & Primaria completa o incompleta & 277 & 60,9 \\
& Secundaria completa o incompleta & 84 & 18,5 \\
& Universitaria completa o incompleta & 9 & 2,0 \\
& Ninguno & 67 & 14,7 \\
& Sin información & 18 & 4,0 \\
Régimen de afiliación & Subsidiado & 210 & 46,2 \\
& Vinculado & 197 & 43,3 \\
Edad al inicio del tratamiento & Contributivo & 43 & 9,5 \\
& Otro & 5 & 1,1 \\
& De 20 hasta 35 años & 83 & 18,2 \\
& $>35$ hasta 50 años & 209 & 45,9 \\
& $>50$ hasta 65 años & 120 & 26,4 \\
\end{tabular}


Cuadro 2. Características clínicas de las pacientes con cáncer de cuello uterino tratadas en el Instituto Nacional de Cancerología, 2000.

\begin{tabular}{|c|c|c|c|}
\hline Variable & Niveles de la variable & $\mathbf{n}$ & $\%$ \\
\hline Tipo histológico & $\begin{array}{l}\text { Escamocelulares } \\
\text { Adenocarcinomas } \\
\text { Oros tipos histológicos }\end{array}$ & $\begin{array}{r}415 \\
26 \\
14\end{array}$ & $\begin{array}{r}91,2 \\
5,7 \\
3,1\end{array}$ \\
\hline Estadio tumoral & $\begin{array}{l}\text { In situ } \\
\text { IA } \\
\text { IB } \\
\text { IIA } \\
\text { IIB } \\
\text { IIIB } \\
\text { IVA } \\
\text { IVB } \\
\text { Sin información }\end{array}$ & $\begin{array}{r}93 \\
2 \\
55 \\
7 \\
74 \\
202 \\
10 \\
8 \\
4\end{array}$ & $\begin{array}{r}20,4 \\
0,4 \\
12,1 \\
1,5 \\
16,3 \\
44,4 \\
2,2 \\
1,8 \\
0,9\end{array}$ \\
\hline Grado de diferenciación & $\begin{array}{l}\text { Bien diferenciado } \\
\text { Moderadamente diferenciado } \\
\text { Mal diferenciado } \\
\text { Indiferenciado } \\
\text { Sin información }\end{array}$ & $\begin{array}{r}40 \\
81 \\
29 \\
3 \\
302\end{array}$ & $\begin{array}{r}8,8 \\
17,8 \\
6,4 \\
0,7 \\
66,4\end{array}$ \\
\hline Tamaño tumoral & $\begin{array}{l}\text { Tumor clínicamente indetectable } \\
\text { Tumores entre } 1 \text { y } 2 \mathrm{~cm} \\
\text { Tumores mayores de } 2 \mathrm{~cm} \text { hasta } 5 \mathrm{~cm} \\
\text { Tumores entre } 5 \text { y } 20 \mathrm{~cm} \\
\text { Sin información }\end{array}$ & $\begin{array}{r}105 \\
2 \\
82 \\
244 \\
22\end{array}$ & $\begin{array}{r}23,1 \\
0,4 \\
18,0 \\
53,6 \\
4,8\end{array}$ \\
\hline
\end{tabular}

los estadios tempranos. Las dos combinaciones más frecuentes de tratamiento fueron la radioterapia y la quimioterapia (13\%), las cuales se emplearon con mayor frecuencia en pacientes en estadio IIB y IIIB. La cirugía y la radioterapia $(8,1 \%)$ se emplearon con mayor frecuencia en pacientes en estadio IB. Vale la pena destacar cinco pacientes con cánceres en estadio II y III, en quienes se hizo tratamiento quirúrgico, a pesar de lo avanzado de su enfermedad (cuadro 3).

\section{Desenlaces clínicos}

De las 455 pacientes elegibles, 303 (66\%) estaban vivas al finalizar el período, $144(31,6 \%)$ habían muerto a causa de la enfermedad y $8(1,8 \%)$ habían muerto por causas no relacionadas con la enfermedad. El tiempo medio de seguimiento para los pacientes que no murieron fue de 3,69 años, con una desviación estándar de 2,58 años. El tiempo medio hasta la muerte para los pacientes que murieron a causa de la enfermedad, fue de 1,50 años con una desviación estándar de 1,29 años. Se observaron algunos valores atípicos para el tiempo hasta la muerte en el grupo de pacientes fallecidas (figura 1).

\section{Análisis de factores de pronóstico}

Considerando que sólo ocho pacientes murieron por causas intercurrentes, se presentan solamente los resultados de supervivencia global, pues las cifras de supervivencia por causa específica no tendrían mayor variación. La probabilidad acumulada de supervivencia global a cinco años fue de 58,8\% y el tiempo medio de supervivencia, de 4,53 años.

Se consideraron el nivel educativo, el régimen de afiliación, el estadio de la enfermedad, el tipo histológico, la edad y el diámetro tumoral, como factores de pronóstico. Las curvas de supervivencia se compararon mediante la prueba de rangos logarítmicos; los resultados se presentan de manera resumida para cada uno de los factores analizados (cuadro 4). Los valores $p$ de la prueba de rango logarítmico demostraron que el nivel educativo, el tamaño del tumor y el estadio tumoral parecen afectar la función de 
Cuadro 3. Características del tratamiento de las pacientes con cáncer de cuello uterino tratadas en el Instituto Nacional de Cancerología, 2000.

\begin{tabular}{|c|c|c|c|c|c|c|c|c|c|c|c|c|}
\hline \multirow{3}{*}{ Estado clínico } & \multicolumn{12}{|c|}{ Tratamientos recibidos } \\
\hline & \multicolumn{2}{|c|}{$\begin{array}{c}\text { Cirugía, } \\
\text { quimioterapia } \\
\text { y radioterapia }\end{array}$} & \multicolumn{2}{|c|}{$\begin{array}{l}\text { Radioterapia } \\
\text { exclusiva }\end{array}$} & \multicolumn{2}{|c|}{$\begin{array}{l}\text { Cirugía } \\
\text { exclusiva }\end{array}$} & \multicolumn{2}{|c|}{$\begin{array}{c}\text { Cirugía y } \\
\text { radioterapia }\end{array}$} & \multicolumn{2}{|c|}{$\begin{array}{l}\text { Radioterapia y } \\
\text { quimioterapia }\end{array}$} & \multicolumn{2}{|c|}{$\begin{array}{c}\text { Sin } \\
\text { información }\end{array}$} \\
\hline & $\mathbf{n}$ & $\%$ & $\mathbf{n}$ & $\%$ & $\mathbf{n}$ & $\%$ & $\mathbf{n}$ & $\%$ & $\mathbf{n}$ & $\%$ & $\mathbf{n}$ & $\%$ \\
\hline $\begin{array}{l}\text { In situ } \\
\text { IA }\end{array}$ & & & 1 & 1,1 & $\begin{array}{r}90 \\
2\end{array}$ & $\begin{array}{r}96,8 \\
100,0\end{array}$ & 2 & 2,2 & & & & \\
\hline IB & 2 & 3,6 & 8 & 14,5 & 13 & 23,6 & 28 & 50,9 & 4 & 7,3 & & \\
\hline IIA & & & 2 & 28,6 & 1 & 14,3 & 3 & 42,9 & 1 & 14,3 & & \\
\hline IIB & & & 43 & 58,1 & & & & & 30 & 40,5 & 1 & 1,4 \\
\hline IIIB & & & 170 & 84,2 & & & 2 & 1,0 & 24 & 11,9 & 6 & 3,0 \\
\hline IVA & & & 9 & 90,0 & & & & & & & 1 & 10,0 \\
\hline IVB & & & 7 & 87,5 & & & & & & & 1 & 12,5 \\
\hline Sin información & & & 2 & 50,0 & & & 2 & 50,0 & & & & \\
\hline
\end{tabular}

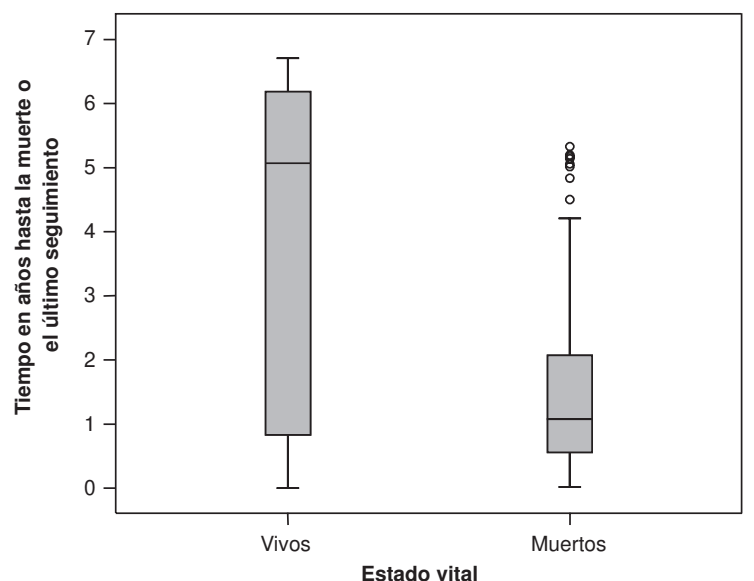

Figura 1. Tiempo en años hasta la muerte o el último seguimiento en pacientes con cáncer de cuello uterino tratadas en el Instituto Nacional de Cancerología, 2000.

supervivencia. Sin embargo, estos hallazgos se deben analizar libres de la confusión que podrían introducir los demás factores de pronóstico a través de un modelo multivariado.

Los resultados del modelo multivariado definitivo de regresión de Cox demostraron que la única variable que afectó la función de riesgo significativamente fue el estadio clínico al momento del diagnóstico (figura 2). Se encontró que el peligro de muerte es cerca de cuatro veces mayor para los pacientes con estadio clínicos I y II, cuando se comparan con pacientes con tumores in situ. Sin embargo, los intervalos de confianza de la razón de peligro no fueron significativos, lo cual se atribuyó a que las diferencias de la supervivencia en estos grupos no son muy grandes y la potencia del estudio no permitió detectarlas. El riesgo de morir se incrementó en 6,2 veces para las pacientes con estadio clínico III, cuando se compararon con aquéllas con tumores in situ. El riesgo de muerte se incrementó hasta en 26,8 veces para las pacientes con estadio IV, cuando se compararon con aquéllas con tumores in situ. Los factores que previamente se habían identificado como posibles factores de pronóstico (tamaño tumoral y nivel educativo) perdieron su significancia en el modelo multivariable (cuadro 5).

\section{Discusión}

En Colombia, los estudios de supervivencia en pacientes con cáncer de cuello uterino son escasos $y$, en este aspecto, la combinación metodológica de revisión de historias clínicas, seguimiento telefónico y revisión de bases de datos de mortalidad, es una alternativa aplicable a instituciones que tengan un registro institucional de cáncer o de base hospitalaria. Los estudios internacionales hacen uso de esta metodología con buenos resultados $(11,12)$.

El Instituto Nacional de Cancerología maneja un alto volumen de pacientes con cáncer de cuello uterino; sin embargo, la población de pacientes elegibles para el análisis se vio 


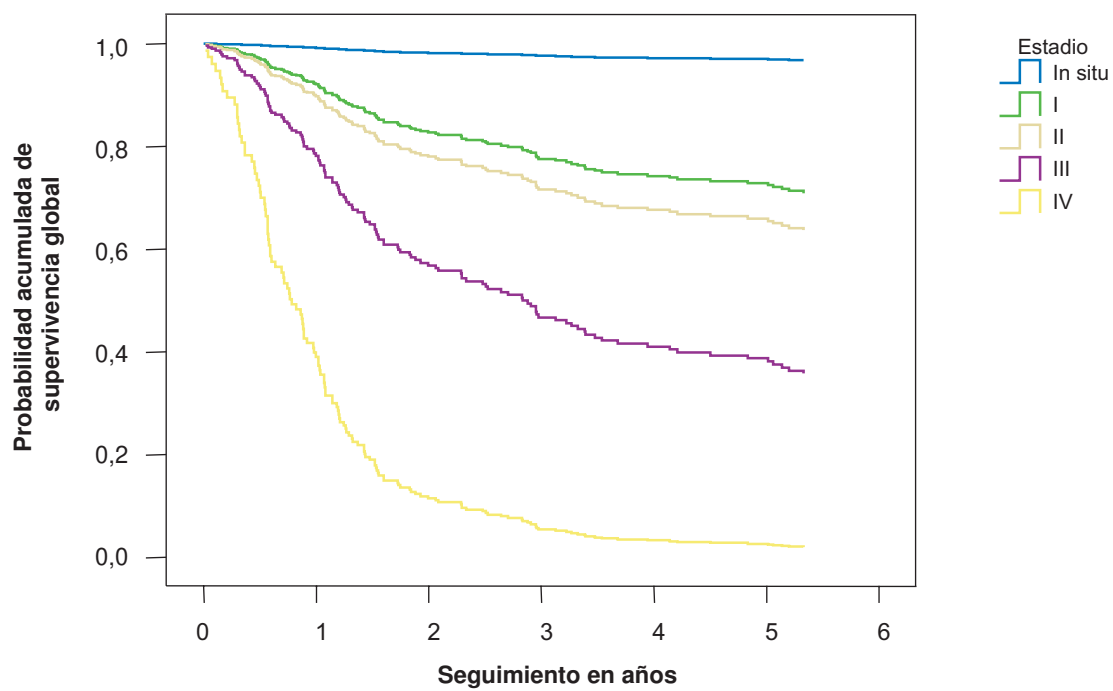

Figura 2. Función de supervivencia global para el estadio obtenida a partir del modelo multivariado de regresión de riesgos proporcionales de Cox, en pacientes con cáncer de cuello uterino tratadas en el Instituto Nacional de Cancerología, 2000

Cuadro 4. Medias de supervivencia en años por factores de pronóstico evaluados en pacientes con cáncer de cuello uterino tratadas en el Instituto Nacional de Cancerología, 2000.

\begin{tabular}{|c|c|c|c|c|}
\hline Variable & Niveles de la variable & $\mathbf{n}$ & $\begin{array}{l}\text { Media de } \\
\text { supervivencia } \\
\text { en años }\end{array}$ & $\mathbf{p}^{*}$ \\
\hline Nivel educativo máximo alcanzado & $\begin{array}{l}\text { Ninguno } \\
\text { Primaria completa o incompleta } \\
\text { Secundaria o más }\end{array}$ & $\begin{array}{r}67 \\
277 \\
93\end{array}$ & $\begin{array}{l}3,9 \\
4,6 \\
5,1\end{array}$ & 0,017 \\
\hline Régimen de afiliación & $\begin{array}{l}\text { Contributivo } \\
\text { Subsidiado } \\
\text { Vinculado }\end{array}$ & $\begin{array}{r}43 \\
210 \\
197\end{array}$ & $\begin{array}{l}5,2 \\
4,6 \\
4,1\end{array}$ & 0,06 \\
\hline Edad al inicio del tratamiento (años) & $\begin{array}{l}\text { De } 20 \text { a } 35 \\
\text { De } 35 \text { a } 50 \\
\text { De } 35 \text { a } 65 \\
\text { Mayores de } 65\end{array}$ & $\begin{array}{r}83 \\
209 \\
120 \\
43\end{array}$ & $\begin{array}{l}5,2 \\
4,3 \\
4,4 \\
4,3\end{array}$ & 0,091 \\
\hline Tipo histológico & $\begin{array}{l}\text { Escamocelulares } \\
\text { Adenocarcinomas } \\
\text { Otros tipos histológicos }\end{array}$ & $\begin{array}{r}415 \\
26 \\
14\end{array}$ & $\begin{array}{l}4,5 \\
5,5 \\
3,6\end{array}$ & 0,159 \\
\hline Estadio tumoral & $\begin{array}{l}\text { In situ } \\
\text { I } \\
\text { II } \\
\text { III } \\
\text { IV }\end{array}$ & $\begin{array}{r}93 \\
57 \\
81 \\
202 \\
18\end{array}$ & $\begin{array}{l}6,6 \\
5,5 \\
5,0 \\
3,4 \\
0,9\end{array}$ & 0,000 \\
\hline Tamaño tumoral & $\begin{array}{l}\text { Tumor clínicamente indetectable } \\
\text { Tumores entre } 1 \text { y } 5 \mathrm{~cm} \\
\text { Tumores entre } 5 \text { y } 20 \mathrm{~cm}\end{array}$ & $\begin{array}{r}105 \\
84 \\
244\end{array}$ & $\begin{array}{l}6,5 \\
4,8 \\
3,6\end{array}$ & 0,000 \\
\hline
\end{tabular}

* Valor p de la prueba de rango logarítmico 
Cuadro 5. Razones de peligro obtenidas mediante el modelo multivariado de regresión de Cox en pacientes con cáncer de cuello uterino tratadas en el Instituto Nacional de Cancerología, 2000.

\begin{tabular}{|c|c|c|c|}
\hline Variable & Niveles de la variable & HR & IC $95 \%$ \\
\hline Edad & $\begin{array}{l}\text { De } 20 \text { a } 35 \text { años } \\
\text { De } 36 \text { a } 50 \text { años } \\
\text { De } 51 \text { a } 65 \text { años } \\
\text { Mayores de } 65 \text { años }\end{array}$ & $\begin{array}{c}1 \\
1,08 \\
1,00 \\
0,95\end{array}$ & $\begin{array}{l}0,61-1,91 \\
0,54-1,87 \\
0,44-2,06\end{array}$ \\
\hline Nivel educativo & $\begin{array}{l}\text { Ninguno } \\
\text { Primaria } \\
\text { Secundaria o universitaria }\end{array}$ & $\begin{array}{c}1 \\
0,62 \\
0,70\end{array}$ & $\begin{array}{l}0,38-1,01 \\
0,37-1,35\end{array}$ \\
\hline Afiliación al SGSSS & $\begin{array}{l}\text { Vinculados } \\
\text { Contributivos } \\
\text { Subsidiados }\end{array}$ & $\begin{array}{c}1 \\
0,47 \\
0,95\end{array}$ & $\begin{array}{l}0,22-1,04 \\
0,66-1,36\end{array}$ \\
\hline Tamaño tumoral & $\begin{array}{l}\text { Tumor clínicamente indetectable } \\
\text { Tumores entre } 1 \text { y } 5 \mathrm{~cm} \\
\text { Tumores mayores de } 5 \mathrm{~cm}\end{array}$ & $\begin{array}{c}1 \\
3,72 \\
4,49\end{array}$ & $\begin{array}{c}0,47-29,21 \\
0,55 \text { a } 36,67\end{array}$ \\
\hline Estadio clínico & $\begin{array}{l}\text { In situ } \\
\text { I } \\
\text { II } \\
\text { III } \\
\text { IV }\end{array}$ & $\begin{array}{c}1 \\
4,18 \\
3,49 \\
7,25 \\
27,86\end{array}$ & $\begin{array}{c}0,38-46,38 \\
0,27-43,83 \\
0,58-90,21 \\
2,04-380,46\end{array}$ \\
\hline Tipo histológico & $\begin{array}{l}\text { Escamocelular } \\
\text { Adenocarcinoma } \\
\text { Otros }\end{array}$ & $\begin{array}{c}1 \\
0,46 \\
1,33\end{array}$ & $\begin{array}{l}0,18-1,19 \\
0,48-3,66\end{array}$ \\
\hline
\end{tabular}

HR: Hazard Ratio (Razón de peligro).

IC 95\%: Intervalo de confianza al 95\%

reducida en $30 \%$. Ello disminuyó la potencia que podría tener el estudio para detectar razones de peligro univariadas superiores 0 iguales a 1,5 con confianza del $95 \%$, desde $88 \%$ hasta $77 \%$, y de esta manera, limitó las posibilidades de explorar factores de pronóstico, más aún cuando se requiere de un análisis multivariado. Se destaca que una pérdida importante de pacientes elegibles estuvo relacionada con la atención fraccionada, la cual se vio reflejada en las que ingresaron con tratamientos previos o en aquéllas que ingresaron y no se trataron por causas ajenas a la institución.

La probabilidad acumulada de supervivencia global para el grupo de pacientes fue de $58,8 \%$. La supervivencia global a cinco años para pacientes con cáncer in situ fue de $98,2 \%$, mientras que la reportada en la literatura es de $100 \%$. Para pacientes con estadios I, la supervivencia global a cinco años fue de $74,5 \%$; la reportada varía entre $75,7 \%$ para estadio IB2 y $97,5 \%$ para estadio IA1. Para pacientes con estadio II, la supervivencia global a cinco años fue de 66,8\%; mientras que la descrita en la literatura varía entre $65,8 \%$ para estadio IIB y $73,4 \%$ para estadio IIA. Los estadios III tuvieron una supervivencia global a cinco años de $37,4 \%$; la informada en la literatura varía entre 39,7\% y $41,5 \%$. Para los estadios IV, la supervivencia global a cinco años fue de $0 \%$, mientras que la reportada en la literatura varía entre 9,3\% para el estadio IVB y $22 \%$ para el estadio IVA (13). Esta única diferencia entre la supervivencia reportada y la observada puede atribuirse al bajo tamaño de muestra de casos en estadio IV, aunque también podría atribuirse a las malas condiciones sociales y de salud en que ingresan las pacientes. No se logró demostrar la relevancia de otros factores diferentes al estadio clínico, en la supervivencia de las pacientes.

Se destaca que la mayor proporción de pacientes que acuden a tratamiento tienen un nivel escolar bajo, están afiliadas al régimen subsidiado o vinculado (población vulnerable) y 
están en el rango de edad entre 35 y 50 años. Estas características pueden explicar el alto porcentaje de estadios clínicos avanzados.

Un alto porcentaje de casos correspondieron a población vinculada, a pesar de que las disposiciones legales tienden a promover la afiliación al régimen subsidiado en la población pobre y, al régimen contributivo, en la población con capacidad de pago (14).

La mayoría de las pacientes tenían tumores de tipo escamocelular y 5,7\% correspondía a adenocarcinomas, lo cual es concordante con la literatura, la cual establece que cerca de $85 \%$ de los cánceres de cuello uterino corresponden a carcinomas escamocelulares $(15,16)$.

La literatura reciente ha demostrado que el uso de radioterapia concomitante con la quimioterapia en los estadios I y II, mejora la supervivencia global y libre de enfermedad (17). Sin embargo, este estudio muestra una baja práctica del uso combinado de quimioterapia y radioterapia en pacientes en estos estadios; esto puede obedecer a que estos datos se generaron de manera muy cercana al periodo en el cual se incluyeron las pacientes para este estudio. Se recomienda hacer estudios descriptivos de fechas recientes, en los cuales se evalúe el uso concomitante de quimioterapia y radioterapia en estadios I y II.

Es necesario destacar algunos casos en estadio II y III, en quienes se hizo tratamiento quirúrgico, a pesar de lo avanzado de su enfermedad. Esta terapéutica inusual estuvo relacionada con el tipo histológico de estos tumores, los cuales correspondieron en su mayoría a sarcomas de cuello uterino (18).

A pesar de que en el análisis de Kaplan-Meier se encontraron como factores de pronóstico el régimen de afiliación, el tamaño tumoral y el estadio clínico al ingreso, el modelo multivariado identificó en este estudio al estadio clínico como el único factor significativo de pronóstico en la función de riesgo. Es posible que un tamaño de muestra mayor hubiese mejorado la potencia del estudio y, en consecuencia, hubiese logrado identificar como significativos a estos factores.
En este estudio se logró un seguimiento hasta la muerte o hasta los cinco años de seguimiento de $67 \%$ de los casos con la metodología descrita, lo cual resulta satisfactorio, puesto que es un porcentaje similar al observado en series internacionales de casos.

Vale la pena resaltar que en este estudio se controlaron sesgos de información y medición que son frecuentes en este tipo de metodología. El contar con personal entrenado en metodología de registros institucionales de cáncer y en seguimiento de pacientes con cáncer, disminuyó estos sesgos. El aplicar la metodología de control de calidad empleada para los registros de cáncer, también permitió identificar algunos errores tanto de información como de digitalización y corregirlos en su totalidad.

De los resultados de la experiencia de investigación, se deben tener en cuenta algunos aspectos que permitieron tener un mayor control sobre el trabajo de investigación. El hecho de que la digitalización fuera hecha por el mismo investigador, permitió detectar de forma más precisa y oportuna las inconsistencias, las cuales se resolvieron con una nueva revisión del formato de recolección o de la historia clínica. La falta de conocimiento en tópicos relacionados con el diagnóstico o tratamiento del tumor, se logró superar con el apoyo de un ginecólogo oncólogo y un especialista en radioterapia.

Es importante contar con fuentes de información, como las bases nacionales de datos de mortalidad general, las cuales permiten actualizar el estado vital de un número importante de registros. Sin embargo, la calidad de la información encontrada en la causa básica de defunción no es buena, ya que existen algunas inconsistencias. El cruce de información permitió identificar, en el certificado de defunción, algunos casos en los que el tumor había sido mal identificado como un tumor de otros sitios o de sitios mal definidos.

El contacto telefónico con las pacientes se convierte en un proceso dispendioso por el continuo cambio de vivienda de las pacientes. Entre las pacientes que tienen su residencia habitual fuera de Bogotá fue difícil el contacto, pero, también, se hizo patente que los 
teléfonos consignados en la historia clínica no corresponden, porque la persona ya no vive allí, la línea ha sido desactivada o quienes contestan la llamada afirman no conocer a la persona por la cual se está indagando.

El cumplimiento del tratamiento instaurado en el Instituto Nacional de Cancerología no fue posible, principalmente, en las pacientes residentes fuera de Bogotá, de lo que se derivan la mayoría de las pérdidas en el seguimiento. Esta situación resulta relevante en un cáncer como el de cuello uterino, el cual tiene una reglamentación establecida para su detección temprana dentro del Sistema General de Seguridad Social. Las dificultades mencionadas ameritan una revisión de las causas existentes en la cobertura, oportunidad y autorización de los tratamientos, por parte de las aseguradoras.

Los resultados obtenidos permiten recomendar el desarrollo de estudios en cohortes más grandes y de fechas más recientes, de tal manera que permitan determinar el efecto de la implementación de la quimioterapia y radioterapia concomitantes, y aumentar la potencia del estudio, para así poder identificar otros factores de pronóstico que no fueron significativos en este estudio, probablemente por el tamaño de la muestra.

\section{Agradecimientos}

A Natascha Ortiz, del Instituto Nacional de Cancerología, y Patricia Arce, de Estadísticas Vitales de la Secretaría Distrital de Salud. A William Marzán y Julieth Castro, por su colaboración en el proceso de recolección de la información, y al Grupo Archivo de Historias Clínicas del Instituto Nacional de Cancerología.

\section{Conflicto de intereses}

No existe ninguno.

\section{Financiación}

Este estudio fue financiado con recursos propios de los investigadores.

\section{Referencias}

1. Ferlay J, Bray F, Pisani P, Parkin DM. Globocan 2002 cancer incidence, mortality and prevalence worldwide. IARC CancerBase No. 5, version 2.0. Lyon: IARC Press; 2004.
2. República de Colombia, Sistema Nacional de Salud, Instituto Nacional de Cancerología. Normas técnicas y administrativas. Guía de implantación, detección y control de cáncer de cuello uterino. Bogotá: Imprenta Nacional; 1994.

3. República de Colombia, Ministerio de Salud. Resolución 00412 de 2000 (25 de febrero), por la cual se establecen las actividades, procedimientos e intervenciones de demanda inducida y obligatorio cumplimiento y se adoptan las normas técnicas y guías de atención para el desarrollo de las acciones de protección específica y detección temprana y la atención de enfermedades de interés en salud pública. Diario oficial No. 49.956, 31 de marzo de 2000. Bogotá D.C.: Ministerio de Salud; 2000.

4. República de Colombia, Ministerio de la Protección Social. Política nacional de salud sexual y reproductiva. Bogotá: Ministerio de la Protección Social; 2000. Fecha de consulta: 23 de octubre de 2008. Disponible en: http:// www.minproteccionsocial.gov.co/vbecontent/library/ documents/DocNewsNo15132DocumentNo1893.PDF.

5. Eifel P, Berek J, Markman M. Cancer of the cervix, vagina, and vulva. In: DeVita V, Hellman S, Rosenberg $\mathrm{S}$, editors. Cancer principles and practice of oncology. 8th edition. Philadelphia: Lippincott Williams \& Wilkins; 2008. p. 1496-543.

6. Pérez C, Kavanagh B. Uterine cervix. In: Halperin E, Pérez C, Brady L, editors. Pérez and Brady's principles and practice of radiation oncology. 5th edition. Philadelphia: Lippincott Williams \& Wilkins; 2008. p. 1533-609.

7. Sankaranarayanan R, Madhukar-Budukh A, Rajkumar R. Effective screening programs for cervical cancer in low- and middle-income developing countries. Bull World Health Organ. 2001;79:954-62.

8. Instituto Nacional de Cancerología. Anuario estadístico 2004. Bogotá, D.C.: Instituto Nacional de Cancerología; 2005. Fecha de consulta: 23 de octubre de 2008. Disponible en: http://www.incancerologia.gov. co/documentos/2_9_2006_2_56_34_PM_Páginas\%20 interiores_total.pdf

9. Dos Santos I. Introducción al análisis de la supervivencia. En: Agencia Internacional de Investigación sobre el Cáncer. Epidemiología del cáncer: principios y métodos. Lyon, Francia: Agencia Internacional de Investigación sobre el Cáncer (IARC); 1999. p. 279-93.

10. Jensen OM, Parkin DM, Mac Lennan R, Muir CS, Skeet RG. Análisis de supervivencia. En: Registros de cáncer: principios y métodos. Publicación científica No. 95. Lyon, Francia: Agencia Internacional de Investigación sobre el Cáncer (IARC); 1995. p. 153-72.

11. Lea JS, Sheets EE, Wenham RM, Duska LR, Coleman RL, Miller DS, et al. Stage IIB-IVB cervical adenocarcinoma: prognostic factors and survival. Gynecol Oncol. 2002;84:115-9. 
12. Farley JH, Hickey KW, Carlson JW, Rose GS, Kost ER, Harrison TA. Adenosquamous histology predicts a poor outcome for patients with advanced-stage, but not early-stage, cervical carcinoma.Cancer. 2003;97:2196202.

13. Quinn MA, Benedet JL, Odicino F, Maisonneuve $\mathbf{P}$, Beller U, Creasman WT, et al. Carcinoma of the cervix uteri. FIGO 6th Annual report on the results of treatment in gynecological cancer. Int $\mathrm{J}$ Gynaecol Obstet. 2006;95(Suppl.1):S43-103.

14. Céspedes JE, Jaramillo I, Martínez R, Olaya S, Reynales J, Uribe C, et al. Efectos de la reforma de la seguridad social en salud en Colombia sobre la equidad en el acceso y la utilización de servicios de salud. Rev Salud Pública. 2000;2:145-64.
15. Rubin $\mathbf{P}$, Williams J. Carcinoma de cérvix uterino. En: Rubin P, editor. Oncología clínica: Enfoque multidisciplinario para médicos y estudiantes. Octava edición. Madrid: Elsevier Science; 2003. p. 463-77.

16. Murphy G, Lawrencw W, Lenhard R. Oncología clínica. Manual de la American Cancer Society. Publicación científica No. 559. Washington, D.C.: Organización Panamericana de la Salud; 1996.

17. Green J, Kirwan J, Tierney J, Vale C, Symonds $\mathrm{P}$, Fresco L, et al. Concomitant chemotherapy and radiation therapy for cancer of the uterine cervix. Cochrane Database Syst Rev. 2005;CD002225.

18. Reed N. The management of uterine sarcomas. Clin Oncol. 2008;20:470-78. 